Artículo recibido el 17 de julio de 2020; Aceptado para publicación el 18 de agosto de 2020

\title{
A cultura Chokwe da Lunda Norte (Angola) e o Programa Etnomatemática: diálogos para repensar a educação em tempos de pandemia
}

\author{
La cultura Chokwe de Lunda Norte (Angola) y el Programa de \\ Etnomatemàtica: diálogos para repensar la educación en tiempos de \\ pandemia
}

\section{The Chokwe culture of Lunda Norte (Angola) and the Ethnomathematics Program: dialogues to rethink education in times of pandemic}

\author{
Cristiane Coppe ${ }^{1}$ \\ Carlos Mucuta Santos ${ }^{2}$
}

\begin{abstract}
Resumo
Este artigo, de cunho reflexivo, nasceu a partir de diálogos entre os autores nas sessões de orientação do Programa de Mestrado em Ciências da Educação da Universidade Lueji A'Nkonde, em parceria com a Faculdade de Educação da Universidade de São Paulo (FEUSP).Os diálogos privilegiaram, tanto as teorizações do educador Ubiratan D'Ambrosio, acerca do papel da educação a partir das dimensões do Programa Etnomatemática no sentido de encaminhar a investigação em vertentes teórico-metodológicas, quanto a visão antropológica da cultura Chokwe da comuna de Camaxilo (Lunda Norte/Angola), especificamente acerca da ação comunitária de aprender no chota cha makulwana ${ }^{3}$. Os encontros ao longo da investigação foram tecendo uma proposta metodológica apoiada nesta ação, contribuindo para novas reflexões e epistemologias que podem emergir ao se pensar no papel social/cultural da escola e da educação ao longo do período da pandemia da COVID-19. Diante do exposto, este artigo pretende compartilhar os diálogos entre orientadora e orientando (Chokwe), ao longo do desenvolvimento de uma investigação de mestrado, buscando caminhos nessa cultura para repensar a escola e seu papel no período de pandemia. $\mathrm{O}$ ato de estar no chota cha mahulwana, pode contribuir para a reflexão de uma postura diferenciada como educadores matemáticos, descontruindo práticas tecnicista impostas pelo ensino remoto. Os diálogos, como encontro culturais, contribuíram para repensar os valores do chota que podem ser atribuídos aos projetos de ensino em aulas de matemática, contribuindo para a constituição de saberes não hegemônicos.
\end{abstract}

Palavras-Chave: Programa Etnomatemática; Cultura Chokwe; Valores do chota cha makulwana.

\footnotetext{
${ }^{1}$ Docente do Instituto de Ciências Exatas e Naturais do Pontal da Universidade Federal de Uberlândia (ICENP/UFU). Diretora da Diretoria de Estudos e Pesquisas Afrorraciais da Universidade Federal de Uberlândia. Membro do Grupo de Pesquisa e Estudo em Etnomatemática da Faculdade de Educação da Universidade de São Paulo e do Núcleo de Pesquisas e Estudos em Educação Matemática da UFU. Membro do corpo docente do Programa de Mestrado em Ciências da Educação da Universidade Lueji N’Konde em Lunda Norte/Angola em parceria com a FEUSP. E-mail: criscopp@ufu.br

2 Mestrando do Programa de Mestrado em Ciências da Educação da Universidade Lueji N’Konde em Lunda Norte/Angola.

${ }^{3}$ Chota cha maulwana é considerado como jango (casa) dos anciãos do povo, o epicentro de toda aldeia chokwe, um símbolo da autoridade, da integridade e da unidade dos membros de uma comunidade chokwe.
} 


\begin{abstract}
This article, of a reflective nature, was born from dialogues between the authors in the orientation sessions of the Master's Program in Educational Sciences of Lueji A'Nkonde University, in partnership with the Faculty of Education of the University of São Paulo (FEUSP). The dialogues focused both on the theorizations of the educator Ubiratan D'Ambrosio about the role of education from the dimensions of the Ethnomathematics Program in order to direct research in theoretical-methodological aspects, as well as the anthropological view of the Chokwe culture of the commune of Camaxilo (Lunda Norte/Angola), specifically about the community action of learning in chota cha makulwana. The meetings throughout the investigation were weaving a methodological proposal supported by this action, contributing to new reflections and epistemologies that may emerge when thinking about the social/cultural role of school and education throughout the covid-19 pandemic period. In view of the above, this article intends to share the dialogues between advisor and mentoring (Chokwe), throughout the development of a master's research, seeking ways in this culture to rethink the school and its role in the pandemic period. The act of being in chota cha mahulwana, can contribute to the reflection of a differentiated posture as mathematical educators, deconstructing technical practices imposed by remote teaching. The dialogues, as a cultural meeting, contributed to rethink the values of the chota that can be attributed to teaching projects in mathematics classes, contributing to the constitution of non-hegemonic knowledge.
\end{abstract}

Key-words: Ethnomathematics Program; Chokwe Culture; Values of Chota cha makulwana.

\title{
1. INTRODUÇÃ̃O
}

\section{Que escola queremos?}

Parece que essa pergunta tem um responda lógica, para a maioria da sociedade, visto que ao se pensar nos valores que o contexto escolar foi constituindo, historicamente, suas teorias, normas, regras e costumes tradicionais, herdaram raízes estritamente europeizantes. Talvez, ao se questionar, a maioria dos pais de estudantes, talvez obtenha-se respostas tais como: "uma escola que prepara para o vestibular", "uma escola que proporcione um ensino de qualidade", "uma escola que prepare para uma profissão que dê uma condição financeira tranquila" e tantas outras respostas que compõem a filosofia e os objetivos da maioria dos sistemas de ensino.

No entanto, em um contexto de pandemia, particularmente, no momento atual que se vivencia o total desconhecimento de como lidar com o novo corona vírus que causa a doença COVID19 , tal pergunta, em um primeiro momento, teria como resposta... o silêncio! O silêncio ou a 
Coppe. C. \& Mucuta. C. (2020). A cultura Chokwe da Lunda Norte (Angola) e o Programa Etnomatemática: diálogos para repensar a educação em tempos de pandemia. Revista Latinoamericana de Etnomatemática, 13(1), 276- 292. DOI: 10.22267/relatem.20131.49

pausa também é uma resposta. Queremos uma escola com as respostas apontadas anteriormente, mas não será possível por conta da necessidade de guardarmos o distanciamento social para preservar vidas. Preservar vidas, em um período pandêmico, torna-se a fato mais relevante para ser pautado nas práticas educativas por videoconferências, ensino remoto por tutoriais, redes sociais e outras alternativas que foram sendo repensadas para que o ensino fosse de alguma forma adequado à uma realidade inesperada.

Esse esforço mundial para, de certa forma, vencer os obstáculos evidenciados por uma pandemia, por um lado, deve levar em conta/valorizar o trabalho exaustivo de planejamento que requer do professor para suas novas práticas, por outro lado, deve levar a reflexão de que essa realidade faz emergir ainda mais as desigualdades sociais das populações consideradas mais vulneráveis nos países. As desigualdades sociais no Brasil, já eram evidenciadas antes da pandemia, no entanto, no contexto educacional em que a única possibilidade de acesso ao ensino remoto se dá pelo acesso a redes de internet, constata-se que a proposta educacional oferecida aos estudantes pela maioria dos estados, é insuficiente em todos os níveis de ensino. Os dados revelados pela pandemia não atingem somente a educação. De acordo com o portal do Instituto Geledés de Mulheres negras (https://www.geledes.org.br/) em notícia publicada em 13 de maio de 2020, revelou que em São Paulo, negros têm até 85\% mais de chance de morrer por COVID-19 do que brancos. Estes dados foram divulgados, a partir de pesquisa realizada pelo Observatório COVID-19 e pela prefeitura da cidade de São Paulo. A notícia, dentre outros apontamentos que comprovam as desigualdades do Brasil em uma realidade pandêmica. A pandemia da COVID-19 acaba "escancarando" as desigualdades sociais, marcadas por raça e gênero, por evidenciar que as mulheres negras estão da linha de frente de trabalhos de limpeza, em serviços de enfermagem, dentre outras.

Nas favelas e comunidade urbanas, os aglomerados de casas já é uma realidade complexa, que acrescida da necessidade de distanciamento social em um período pandêmico, gera ainda mais situações de vulnerabilidade, em que a maioria das construções, com um ou dois cômodos, na maioria das vezes, necessita comporta uma família com dez pessoas, por exemplo.

Essa realidade remete a uma volta à pergunta inicial “Que escola queremos?” e, olhando para o contexto educacional brasileiro, acompanha-se movimentos que desconsideram as 
desigualdades acentuadas pela pandemia, mantendo exames padronizados (ENEM, vestibulares, dentre outros) e tentando adequar os conteúdos de um programa pensado para o formato presencial para atividades remotas. Retoma-se uma postura tecnicista da prática docente, como uma ação de retrocesso do processo de ensino e de aprendizagem.

Em um processo de reflexão, a escola que espera-se constituir pela experiência atravessada por uma pandemia, poderia ser aquela que repensasse seu papel na sociedade na formação de crianças e jovens que aprendessem valores culturais e de empatia com o outro. Esta postura extrapola os muros da escola e poderá ganhar lugar no processo de ensino e de aprendizagem para esse período.

A partir dessas palavras iniciais, este artigo pretende compartilhar algumas ideias que surgiram a partir de diálogos entre orientadora e orientando (Chokwe), autores desse trabalho, ao longo do desenvolvimento de uma investigação de mestrado. Buscou-se caminhos nessa cultura para repensar a escola e seu papel na sociedade, tendo o estudo evidenciado que os valores culturais encontrados no chota cha makulwana podem ampliar os sentidos da escola e seu papel nesse período de pandemia, para além de práticas tecnicistas impostas pelo ensino remoto.

\section{O "ESTAR NO CHOTA", O PROGRAMA ETNOMATEMÁTICA E A ESCOLA}

A ação pedagógico-cultural de estar no chota cha makulwana é uma prática do povo Chokwe localizado da região da Lunda Norte em Angola no continente africano. A província de Lunda Norte divide-se em 10 municípios (Cambulo, Capenda- Camulemba, Caungula, Chitato, Cuango, Cuilo, Lóvua, Lubalo, Lucapa e Xá-Muteba). Camaxilo é única comuna do município do Caungula. Nessa região as tradições culturais ainda são muito presentes nas práticas do povo Chokwe, que segundo Coppe e Santos (2019),

acredita-se que sejam imigrantes bantus oriundo do Sahara (África do norte), que inicialmente ocuparam a região do Níger e Ubangi (região dos grandes lagos) e seguidamente a África Austral. Atualmente, o povo Chokwe encontra-se na Zâmbia, Moçambique, República Democrática do Congo (RDC) e em Angola. Em Angola, o povo Chokwe, maioritariamente, ocupa a região leste (Moxico e Lundas) que envolve os nascentes e cursos médio dos rios: Cassai, Luachimo, Chicapa, Luangue, Lovua, Chihumbue, Luchico, Cuilo, Lubale, Luembe, Cuengo, Uhamba e Cuango. Também encontra-se Chokwe nas províncias de Bié, Kuando Kubango, Cunene e Malange. Em 
Coppe. C. \& Mucuta. C. (2020). A cultura Chokwe da Lunda Norte (Angola) e o Programa Etnomatemática: diálogos para repensar a educação em tempos de pandemia. Revista Latinoamericana de Etnomatemática, 13(1), 276- 292. DOI: 10.22267/relatem.20131.49

Luanda (capital de Angola) os Chokwe estão principalmente no Município da Viana e em Rocha Pinto. (COPPE \& SANTOS, 2019, p. 188)

O povo Chokwe da comuna do Camaxilo faz, explica, utiliza e interpreta as unidades de medidas no seu dia a dia tendo como base o Nzongo. Tal evidência, contribui para se pensar em uma proposta de ensino que possa minimizar a distância entre as unidades padronizadas e não-padronizadas na região de Camaxilo em que a maioria dos estudantes são do povo Chokwe.

Neste contexto, o segundo autor deste artigo, além de mestrando e pertencente ao povo Chokwe da comuna de Camaxilo em Angola, foi instruído no chota cha makulwana (Figura 1), e, ao longo da pesquisa, no desafio de pesquisar sua própria cultura, especificamente, a unidade de medidas Nzongo, problematizou a possibilidade de se pensar em um educação pautada nos valores ensinados no chota. Tal possibilidade abriu caminhos para uma prática docente que pode considerar as raízes culturais de estudantes do Camaxilo ao propor a inclusão do $\mathrm{Nzongo}^{4}$ no ensino de grandezas e medidas.

A busca por uma problematização sobre a linguagem matemática Chokwe e a possibilidade de valores culturais no sistema de ensino em Angola, buscou inspiração ao se pensar na ampliação da Lei 17/16, de 07 de outubro da Lei de Bases do Sistema de Educação e Ensino em vigor em Angola que apresenta como um de seus objetivos de ensino "[...] Garantir a valorização das línguas nacionais, da cultura local e da cultura nacional”. Considerando que o povo Chokwe tem uma cultura artística e matemática, que precisa ser resgatada por meio de investigações, propôs-se a valorizar a cultura local do povo chokwe do Camaxilo pesquisando sobre o Nzongo e suas sub-unidades.

Para além do Nzongo como unidade de medidas, que não será o foco principal deste artigo, os valores Chokwe aprendidos no chota cha makulwana, podem inspirar novos valores para a escola, principalmente em tempos sombrios na história da humanidade, tal como as pandemias. Essa possibilidade foi sendo tecida nas sessões de orientações em um processo

\footnotetext{
${ }^{4}$ Para compreender um pouco mais o contexto da pesquisa consultar o artigo de COPPE, C.;SANTOS. C.M., O contexto cultural e o contexto escolar em Angola: conhecendo Nzongo - unidade de medida do povo Chokwe na comuna de Camaxilo no endereço: http://histemat.com.br/index.php/HISTEMAT/article/view/278
} 
dialógico em que a cultura local foi se inserindo no contexto da investigação entre orientando e orientadora, autores deste artigo.

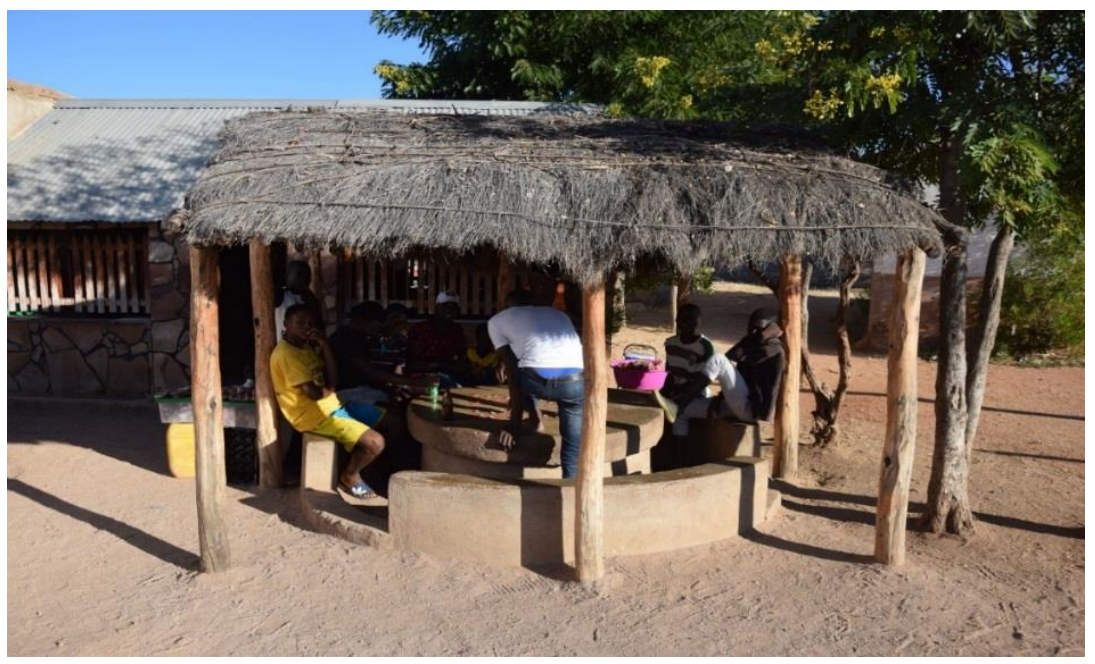

Figura 1 - Chota cha makulwama na comuna de Camaxilo

Fonte: Arquivo pessoal dos autores

Muito tempo antes da ocupação colonial portuguesa, de acordo com conhecimentos Chkowe do orientando, as populações do Camaxilo estiveram já estabelecidas no Mussumb' wa Chiyekel' (aldeia real), organizadas em famílias, sendo o meio educativo exclusivamente a oralidade exercida no chota cha makulwana. Atualmente tal fato ainda ocorre, podendo-se notar que a educação de jovens educados no chota cha makulwana é diferente dos jovens das cidades.

O chota cha makulwana (jango) é/era o local de instrução local que, diariamente abria as portas para convívios humanitários, familiares e amizades. Pela oralidade, eram transmitidas experiências da vida, habilidades e capacidades ideológicas, físicas, religiosas, morais, dentre outras. Isto, mostra a grande necessidade da interculturalidade, pois os saberes ditos tradicionais são tão necessários como os conhecimentos oriundos das novas formas tecnológicas.

Estar no chota cha makulwana como metodologia, ao longo da pesquisa, para a produção de conhecimento conduziu-se, no processo de orientação, chegando ao entendimento de que o 
Coppe. C. \& Mucuta. C. (2020). A cultura Chokwe da Lunda Norte (Angola) e o Programa Etnomatemática: diálogos para repensar a educação em tempos de pandemia. Revista Latinoamericana de Etnomatemática, 13(1), 276- 292. DOI: 10.22267/relatem.20131.49

chota cha makulwana é um lugar que consiste, principalmente, em cinco valores culturais que poderiam nos auxiliar na construção de um pensamento diferente para a escola e para a prática em sala de aula. E por que não dizer, para tempos de pandemia?

A partir das vivências do segundo autor e pelas próprias instruções recebidas por ele na comuna de Camaxilo, alguns elementos caracterizando o ação de "estar no chota cha makulwana":

- Resulta no reconhecimento da igualdade dos caminhos para se chegar ao conhecimento. Sem ofuscar os avanços das ciências, admite-se que conhecimento é obtido por tradição popular, tendo como exemplo no contexto do cotidiano Chkowe a unidade nzongo.

- É aprender com os mais velhos. No chota cha makulwana aprende-se para a vida; os maisvelhos (anciãos) são responsáveis pela transmissão de valores, habilidades e valores humanos para vida as novas gerações. Por exemplo, na luta pela sobrevivência, no chota, os homens aprendem como recompensar a caça que não foi produtiva, usando armadilhas como mukunhi, chifika, etc.

- É transmitir e receber valores. O respeito pelas pessoas e a valorização da vida humana são valores do povo chokwe desde antiguidade. O chota cha makulwana é frequentado, de forma geral, por pessoas do sexo masculino anciãos circuncidados, pois o incircunciso não tem direito ao chota, por ser considerado inapto para absorção de khata ja ku mukanda (códigos vitais da cultura chokwe). Os contos e provérbios do rei Mwene Muachissengue são ensinados e interpretados no chota cha makulwana, o que é uma herança cultural, pois há diversas comunidade Chkowe atualmente que não possuem chota e as tradições orais vão se perdendo. O papel educativo do chota cha makulwana é abrangente na medida em que, todos podem aprender de qualquer mais-velhos presente no chota. É diferente da educação familiar, que é particularidade de cada família. No chota cha makulwana, o conhecimento é exposto e é apropriado por observação e audição atenciosas de cada observador e ouvinte.

- É aprender a receber visitas na aldeia. Uma dos valores aprendidos no chota cha makulwana é a capacidade de interagir com pessoas de culturais diferentes, por meio de uma

\footnotetext{
${ }^{5}$ Khata ja ku mukanda: linguagem e sinais de comunicação exclusivas das pessoas chokwe circuncidadas.
} 
linguagem coloquial, manifestando a capacidade de experienciar a cultura de outras pessoas e ser compreensivo, interessado e curioso para com o visitante e após a visita há a escrita do mujimbo /lusango (relatório síntese da viagem).

Observa-se que, no contexto escolar de Angola, o mujimbo não teria o mesmo valor cultural, pois poderia ser elaborado por um estudante que não foi instruído no chota. O mujimbo tem um papel relevante na socialização das pessoas. Por ele, o visitante identifica-se e apresenta a sua missão e um membro da comunidade apresenta a ele, as condições de recepção, o estado e acontecimentos de relevância da aldeia ou da região. É importante saber contar mujimbo (lusango), pois é simbolo de maturidade e conhecimento, assim como a demonstração de capacidade para encontros interculturalidade. Ser competente interculturalmente falando, implica ter conhecimento cultural e também atitudes, crenças, valores e aptidões interpessoais.

Nos bairros e aldeias Chokwe, a hospitalidade é uma virtude da oralidade transmitida por Mwene Muachissengue, rei dos Chokwe. Neste contexto, constata-se que a interculturalidade exercida no chota cha makulwana expressa nos contos do rei Mwene Muachissengue, como “zumbula ngueji, mba umone cha mbata ngueji", cuja tradução literal é: "só quem acolhe o visitante, saberá o que leva o visitante"; tem como fundamento, o aceitar a condição de outras pessoas e aprender delas o possível para o nosso próprio desenvolvimento.

- É promover a paz entre as pessoas. A resolução de problemas e a pacificação de espírito é um dos papeis preponderantes do chota cha makulwana. No chota são trazidas todas as situações de desentendimentos interpessoais, intercomunitárias e interregionais. As pessoas se reúnem de forma circular. Os conflitos da comunidades tais como, desentendimentos passionais, problemas econômicos, familiares, entre a vizinhaça, divisão de terra, dentre outros, quando levados ao chota, necessariamente, resultam na recuperação do entendimento e paz entre os envolvidos. Mesmo que o fórum no interior do chota, decida pela culpa de uma pessoa, a pessoa central do chota que é rei da aldeia, que tem o respeito de todos, decide pela preservação da vida e ao perdão, retomando a paz entre uma situação conflituosa. Esta ação tem fundamento no provérbio do rei Mwene Muachissengue: "huma dia mwata, dia tola mvula" e "mu chota a mwangamo meya, hi manhinga ko" (na presença do rei não pode haver derramamento de sangue). 
Coppe. C. \& Mucuta. C. (2020). A cultura Chokwe da Lunda Norte (Angola) e o Programa Etnomatemática: diálogos para repensar a educação em tempos de pandemia. Revista Latinoamericana de Etnomatemática, 13(1), 276- 292. DOI: 10.22267/relatem.20131.49

As antropologias e valores dos povos africanos sempre revelam caminhos para o aprendizado em meio a oralidade. A aproximação com os valores do chota, nos diálogos de orientação ao longo da pesquisa, revelando a igualdade de caminhos para se chegar ao conhecimento; o aprender com os mais velhos; o transmitir e receber valores, o aprender a receber visitas e a promoção da paz entre as pessoas, compõem um conjunto de elementos que remete à Ética da Diversidade, proposta por D`Ambrosio (1997) cujos princípios básicos são: respeito pelo outro e todas as suas diferenças, solidariedade com o outro na satisfação de necessidades de sobrevivência e transcendência e cooperação com o outro na preservação do patrimônio natural e cultural comum (D’AMBROSIO, 1997, p. 58).

De acordo com Abreu (2017):

Torna-se uma necessidade que os valores, a ética e a moralidade se associem aos conhecimentos ensinados na escola. Vivemos num mundo onde há muita produção técnica de saberes e pouca reflexão com os impactos no planeta da implementação desses saberes. Nossa sociedade não carece de profissionais capacitados, necessita de profissionais comprometidos com a existência do outro e com a manutenção da vida humana de forma digna, do mesmo modo que a de milhões de outras espécies que habitam neste planeta. (ABREU, 2017, p.81)

Nessa perspectiva, encaixam-se as dimensões do Programa Etnomatemática que valorizam as vertentes que podem fazer interfaces entre passado e presente. A dimensão pedagógica do Programa Etnomatemática, aliada aos valores do "estar no chota" aprendendo da filosofia e da antropologia tradicional Chkowe, realça as considerações de D’Ambrosio (2001) ao afirmar que,

um enfoque etnomatemático sempre está ligado a uma questão maior, de natureza ambiental ou de produção, e a etnomatemática raramente se apresenta desvinculada de outras manifestações culturais, tais como arte e religião. A etnomatemática se enquadra perfeitamente numa concepção multicultural e holística de educação. O encontro intercultural gera conflitos que só poderão ser resolvidos a partir de uma ética que resulta do indivíduo conhecer-se e conhecer sua cultura e respeitar a cultura do outro. O respeito virá do conhecimento. De outra maneira, o comportamento revelará arrogância, superioridade e prepotência, o que resulta, inevitavelmente, em confronto e violência (D’AMBROSIO, 2001, p. 44-45).

No contexto da pesquisa, com a proposta de inserir o Nzongo no ensino das grandezas e medidas na comuna de Camaxilo, a Etnomatemática possui elementos que comungam com os princípios da lei 17/16, Lei de Bases do Sistema de Educação e Ensino em Angola (o reconhecimento dos saberes /valores que compõem a cultura local dos estudantes). Assim 
como a Lei 17/16, a Etnomatemática, aliada aos saberes aprendidos no chota, propõem o trabalho com os conhecimentos do contexto sociocultural, geralmente, não formalizados, muitas vezes nem aceitos no meio acadêmico-científico, mas que são conhecimentos emergentes do cotidiano e da oralidade de um povo, tal como ocorre com o povo Chkowe. Como os conhecimentos culturais acabam caminhando de forma disassociada do contexto escolar, pois as escolas, na maioria das vezes, ignoram os saberes de tradição oral e/ou do cotidiano, o Programa Etnomatemática, de certa forma, vem contribuindo para ouvir diversas vozes, possibilitando a propagação de sua cultura, identidade, saberes e produção do conhecimento, propiciando-lhes reconhecimento e valorização.

Tal como aponta Boaventura de Sousa Santos e Maria Paula Meneses na obra Epistemologias do Sul, sobre o resgate de modelos epistemológicos, hoje desconsiderados pela soberania epistêmica das ciências,

[...] ao longo da modernidade, a produção do conhecimento científico foi configurada por um único modelo epistemológico, como se o mundo fosse monocultural, que descontextualizou o conhecimento e impediu a emergência de outras formas de saber não redutíveis a esse paradigma. Assiste-se, assim, a uma espécie de epistemicídio, ou seja, a destruição de algumas formas de saber locais, à inferiorização de outros, desperdiçando-se, em nome dos desígnios do colonialismo, a riqueza de perspectivas presente na diversidade cultural e nas multifacetadas visões do mundo por elas protagonizadas. (SANTOS; MENESES, 2009, p. 7).

Nesse sentido, a escola, inspirada pelo conhecimento e valores do chota, pode propiciar um repensar dos métodos e formas, do tradicionalismo e formalismo, do capitalismo e conteudismo e do conservadorismo impregnados nas práticas pedagógicas. Este repensar neste período de pandemia da COVID-19, por um lado, desmonta os padrões e normas, quase que imutáveis, dos sistemas de ensino urbanos, contradizendo antigas falas direcionadas por professores e coordenadores pedagógicos tais como "não pode usar celular na sala de aula", "não pode vir para a escola de chinelo", "tem que ficar com uma postura correta na carteira", dentre outras regras que circulam nos regimentos internos escolares.

Por outro lado, o ensino remoto aprissionou a prática docente em um movimento de retrocesso frente a uma perspectiva tecnista. Tal constatação, evidencia-se ainda mais, no processo de ensino e de aprendizagem em matemática, no qual têm-se privilegiado resolução de exercícios como atividade assíncrona e aula extremamente expositivas por meio de uma 
Coppe. C. \& Mucuta. C. (2020). A cultura Chokwe da Lunda Norte (Angola) e o Programa Etnomatemática: diálogos para repensar a educação em tempos de pandemia. Revista Latinoamericana de Etnomatemática, 13(1), 276- 292. DOI: 10.22267/relatem.20131.49

plataforma digital com atividade síncrona. Essas ações vem se constituindo com uma realidade para uma pequena parte da população de estudantes no Brasil que possuem acesso a internet. A exclusão de muitos estudantes contrapõem-se ao que se acredita ser uma postura ideal como metodologia de trabalho frente à pandemia da COVID-19 - o estar no chota!

O tempo que a escola se preocupa em suas reuniões pedagógicas, ocupando lugar de valores que deveriam ser ensinados, ao invés de regras e normas, poderia ser aproveitado para incorporar diálogos acerca da Ética da Diversidade (respeito, solidariedade e cooperação com o outro) e dos ensinamentos do "estar no chota (a igualdade de caminhos para se chegar ao conhecimento; o aprender com os mais velhos; o transmitir e receber valores, o aprender a receber visitas e a promoção da paz entre as pessoas).

Essa mudança de postura e o repensar do papel da escola, como uma ação contínua de constante aprendizado, traria para além dos conteúdos, prioritariamente de pensamento eurocêntrico, outros valores para o contexto escolar e para a formação de seus estudantes. Em tempos de pandemia, esses valores seriam ressaltados e poderiam possibilitar diálogos em que os grupos em situações mais vulneráveis à doença, tivessem apoio em um movimento intercultural, promovendo a empatia.

\section{CONSIDERAÇÕES}

A partir dos diálogos que foram sendo tecidos nas sessões de orientação da investigação, pode-se pensar que, a experiência de compreender os sentidos do aprender na ação de "estar no chota chamakulwana”, leva a repensar a escola e seus sentidos, principalmente frente a realidade da pandemia provocada pela COVID-19.

Aprender que é possível a igualdade de caminhos para se chegar ao conhecimento; aprender com os mais velhos; transmitir e receber valores, aprender a receber visitas e a promoção da paz entre as pessoas, valores do povo Chkowe aprendidos no chota, aliados à Ética da Diversidade (respeito pelo outro,solidariedade com o outro e cooperação com o outro) proposta pelo educador matemática Ubiratan D’Ambrosio, pode ajudar a responder a pergunta inicial “Que escola queremos?” para os tempos de pandemia. Uma escola que possibilite a experiência de se colocar no lugar do outro (empatia) que permita construir 
novos caminhos para serem trilhados, buscando a aproximação do contexto escolar com a realidade vivenciada pelos estudantes.

O desafio para a comunidade de educadores matemáticos se põe, nesse período de pandemia, como um movimento de reflexão, que possa deslocar a pergunta "Que escola queremos"? para outras novas perguntas, tais como: "Que escola podemos pensar para a pandemia, como educadores matemáticos?”, “Que ensino podemos proporcionar para nossos estudantes, nesse período, que contraponha-se ao ensino meramente tecnicista?", "Que escola e que ensino podemos constituir coletivamente após esse período que fomos atravessados pela pandemia?"

Muitas respostas podem passar pela cabeça do leitor. No entanto, esse artigo de caráter reflexivo, o convida para pensar em possibilidades a partir dos elementos culturais de aprendizado evidenciados pela ação de "estar no chota". A igualdade de caminhos para se chegar ao conhecimento, pode ser compreendida como ações de solidariedade, promovida pelo educador, juntamente com seus estudantes e a gestão escolar, em favor de estudantes que perderam o acesso ao ensino. Pode-se encaminhar conteúdos do programa de matemática, juntamente com mensagens escritas de estudante para estudante, que valorizam a ética da diversidade ou mesmo colaborem com instruções para a prevenção da doença, fazendo chegar em regiões distantes da cidade, tal como a zona rural, comunidades indígenas e quilombolas. A realidade de muitas escolas públicas brasileiras evidenciar que há a oferta de material impresso referente aos conteúdos, no entanto as famílias precisam ir até a escola buscá-lo.

Aprender com os mais velhos, nesse período de pandemia, é exercitar a escuta, visto que a oralidade é um valor importante da cultura Chkowe. O educador (matemático) pode propor uma atividade de interação, mesmo que virtual, para ouvir dos mais velhos na família dos seus estudantes, história de vida de como a matemática compõe/compôs suas práticas cotidianas, valorizando seus saberes. Essa proposta poderá além de valorizar o aprendizado com os mais velhos, tirar o idoso da condição de isolamento total, em função do risco eminente de contrair a COVID-19.

Manter as tradições orais, compreendo discursos não hegemônicos da história da matemática de diversas civilizações e culturas, pode contribuir para a descolonização do conhecimento 
Coppe. C. \& Mucuta. C. (2020). A cultura Chokwe da Lunda Norte (Angola) e o Programa Etnomatemática: diálogos para repensar a educação em tempos de pandemia. Revista Latinoamericana de Etnomatemática, 13(1), 276- 292. DOI: 10.22267/relatem.20131.49

escolar, exercitando o elemento cultural de transmitir e receber valores nesse processo entre passado e presente, contrapondo-se, subversivamente, ao ensino remoto tecnicista, imposto na pandemia como um modelo pedagógico.

Outra ação que pode contribuir como uma forma de resistência ao modelo remoto de ensino aplica-se às avaliações. Pode-se tomar como modelo o mujimbo (relatório) de visitas do chota, podendo estabelecer aproximações com discursos de personalidades no campo da educação matemática ou de outros campos do saber, que realizam movimentos acerca de diversos temas transversais que estão emergindo no período de pandemia, podendo ser um jornalista, um filósofo, um educador, um ativista de movimentos sociais, um representante de uma comunidade, dentre outros. O mujimbo funcionaria como um documento de reconhecimento de que a cidadania em tempos de pandemia deve ser exercida mais veementemente.

Por fim, promover a paz entre as pessoas, na solução de problemas, pode auxiliar o educador matemático a repensar suas práticas, a partir da concepção da matemática para a justiça social. Esse caminho alia-se as ideias propostas pelo educador matemático americano Eric Gutestein em que considera que ler e escrever o mundo com a matemática significa, essencialmente, que os estudantes devem usar e aprender matemática para estudar sua realidade social, para que possam ter uma compreensão mais profunda do mundo e possam estar preparados para mudá-lo, assim como acharem conveniente. (MOURA \& FAUSTINO, 2017).

Essas considerações ganham vida pelos caminhos do Programa Etnomatemática ao compreender a educação (matemática) numa concepção multicultural, holística e pautada no respeito que vem a partir do conhecimento. E no que se refere à comunidade de educadores matemáticos no Brasil, há uma sutil mobilização com algumas ações de resistência em relação ao ensino remoto imposto. Destaca-se o documento "Diálogo sobre o Plano de Estudo Tutorado da Secretaria Estadual de Educação de Minas Gerais”, discutido e socializado pela Sociedade Brasileira de Educação Matemática do estado. A intenção desse movimento foi o de salientar que as medidas propostas para minimizar os efeitos negativos do ensino remoto, necessitam de um amplo debate entres os pares e com instituições de ensino comprometidas 
com a educação pública. $\mathrm{O}$ documento ${ }^{6}$ aponta além de problemas conceituais, situações em que as propostas pedagógicas desconsideram o contexto sócio-cultural do estudante, ao exigir tarefas que requer acesso à internet ágil e outros elementos que resultam na exclusão.

Outra mobilização que cabe ressaltar é a doação a instituições assistenciais, por parte de discentes que realizam suas pesquisas de mestrado e doutorado em um Programa de PósGraduação em Ensino de Ciências e Matemática e que organizaram o evento científico XXIII Encontro Brasileiro de Estudantes de Pós-Graduação em Educação Matemática EBRAPEM $^{7}$. Foram doados alimentos, materiais de limpeza e higiene, equipamentos de segurança contra a doença, dentre outros. Os produtos foram custeados pelo saldo financeiro do evento que foi realizado no ano de 2019 na cidade de São Paulo.

Essas tímidas ações possibilita a reflexão de que, tanto no contexto da sala de aula, quanto no contexto da pesquisa, o campo da Educação Matemática pode mobilizar-se com ações de resistência aos modelos pedagógicos impostos para o ensino remoto e sair do conforto da condição de pesquisador de mestrado ou doutorado e levantar ações de empatia durante a pandemia. E que os elementos culturais aprendidos no ato de estar no chota cha makulwana pode nos inspirar/ensinar com outros valores e outros saberes estabelecendo pontes entre a desafiante relação de se pensar a matemática e a sociedade.

\footnotetext{
${ }^{6}$ Acesse o documento no seguinte endereço: http://www.sbembrasil.org.br/files/analise_plano_mg.pdf ${ }^{7}$ Para mais informações acesse http://www.sbembrasil.org.br/sbembrasil/index.php/noticias/930-discentesorganizadores-do-xxiii-ebrapem-realizam-doacoes-para-instituicoes-assistenciais.
} 
Coppe. C. \& Mucuta. C. (2020). A cultura Chokwe da Lunda Norte (Angola) e o Programa Etnomatemática: diálogos para repensar a educação em tempos de pandemia. Revista Latinoamericana de Etnomatemática, 13(1), 276- 292. DOI: 10.22267/relatem.20131.49

\section{REFERÊNCIAS}

Abreu, R.G. (2017). Uma história oral da Etnomatemática: caminhos para a dimensão educacional (Dissertação de mestrado). Faculdade de Educação da Universidade de

São Paulo Recuperado de
https://www.teses.usp.br/teses/disponiveis/48/48134/tde- $\quad 16102017-122220 /$ ptbr.phphttps://www.teses.usp.br/teses/disponiveis/48/48134/tde- 16102017-122220/ptbr.php

D`Ambrosio, U. (1997). Transdisciplinaridade. São Paulo: Palas Athenas.

D`Ambrosio, U. (2001). Etnomatemática: elo entre as tradições e a modernidade. Belo Horizonte: Autêntica.

Coppe, C. \& Santos. C. (2019). O contexto cultural e o contexto escolar em Angola: conhecendo Nzongo - unidade de medida do povo Chokwe na comuna de Camaxilo. Revista de História da Educação Matemática, 5(2). 186-202. Recuperado de http://histemat.com.br/index.php/HISTEMAT/article/view/278

Moura, A., Faustino, A. (2017). Eric Gutstein e a leitura e escrita do mundo com a matemática. RPEM, Campo Mourão.

Santos, B., \& Meneses, M. (2009). Epistemologia do Sul. São Paulo: Cortez. 